\title{
TOWARDS AUTOMATED QUALITY ASSESSMENT OF CONSTRUCTION ELEMENTS
}

\author{
Alexander Braun ${ }^{1}$, Frédéric Bosché2 ${ }^{2}$ and André Borrmann ${ }^{3}$
}

\section{Background and Identification of Problem / Knowledge GaP}

Construction progress monitoring has gained increasing interest in the recent decade due to the implementation of Building Information Modeling and affordable and efficient Reality Capture technologies. The latter include Laser scanning (Bosché and Haas, 2008) as well as photogrammetric methods (Golparvar-Fard et al., 2009). Scan-vs-BIM methods allow an as-planned vs. as-built comparison to make inferences on the presence of individual construction elements. With the incorporation of 4D data, statements on the construction progress are possible (Turkan et al., 2012). However, point clouds do not always provide sufficient or adequate information for quality assessment. Thus, recent research has been focussing on image-based methods and deep learning to solve this problem. For example, several researchers effectively detect cracks in asphalt or concrete elements using images instead of 3D point clouds (NhatDuc, Nguyen and Tran, 2018).

The authors propose to incorporate photogrammetry-based Scan-vs-BIM workflows with image-based processing enhancements to make detailed inferences on construction quality as well as providing continuous and semanticallyclassified image data for QA personnel.

\section{Research Aim And Methodology}

The presented research aims at the extension of currently established, point cloud-based construction progress monitoring workflows to facilitate quality control during construction. For this purpose, photogrammetric point clouds and the underlying images are used as a basis. The Structure from Motion (SfM) process estimates the initial camera positions for all points of view. is the point cloud is then alignedwith the corresponding digital building model using wellestablished site (geo)referencing surveying techniques. With the knowledge of each element and the position of the cameras, the $3 \mathrm{D}$ elements can be back-projected into the 2D plane of all images (Braun and Borrmann, 2019). For example, Figure 1 shows a sample of a back-projected column.

It is crucial to mention that not all elements should be back-projected into the image plane. Instead, only those elements that were detected as built in a previous point cloud as-planned vs. as-built detection are back-projected.

After completing the back-projection for all detected elements at all times of observation, a list of all images that contain visual data about any given construction element is obtained automatically (see Figure 2). Additionally, the as-planned vs. as-built comparison returns all

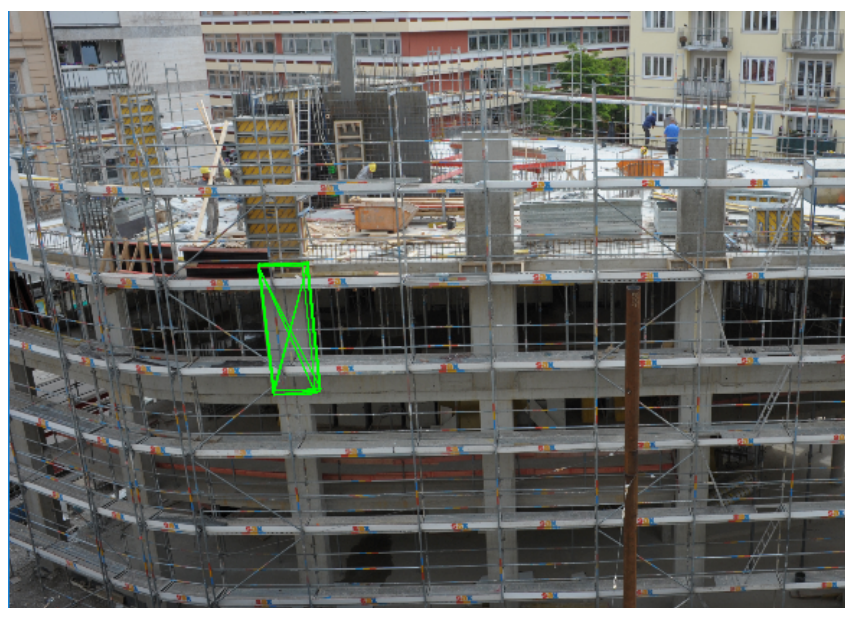

Figure 1: Projected 3D geometry of a column into the

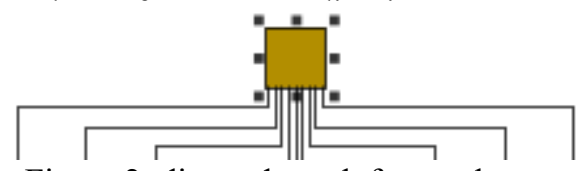

Figure 2: directed graph for an element and all images that it is visible on $3 \mathrm{D}$ points that were matched to this given object, and these points can also be back-projected to the image that they were reconstructed from. This allow identifying the image, that reconstructed most points, which can be seen as the image that shows an element from the best point of view.

In the next step, the 'as-built' textures from the pictures can be projected onto the geometry of the digital model and these textures can be analysed to further assess quality.

Research Associate, Chair of Computational Modeling and Simulation, Technical University of Munich, alex.braun@tum.de Senior Lecturer, School of Engineering, University of Edinburgh, f.bosche@ed.ac.uk

Professor, Chair of Computational Modeling and Simulation, Technical University of Munich, andre.borrmann@tum.de 


\section{RESEARCH FINDINGS}

The proposed methods were tested on real-world construction sites. Figure 3 shows the resulting relations between all detected elements and the images taken during observation. With the help of this graph, all photos that show a specific element can be quickly identified. This provides an effective means to organise semantically the acquired pictures, which is of value to construction professional when they seek to retrieve visual information about anyconstruciton element.

Quality Assessment can be either done manually on these provided pictures, but also in automated ways, e.g., by detecting cracks on the surface of these elements using now increasingly popular (and powerful) deep learning techniques.

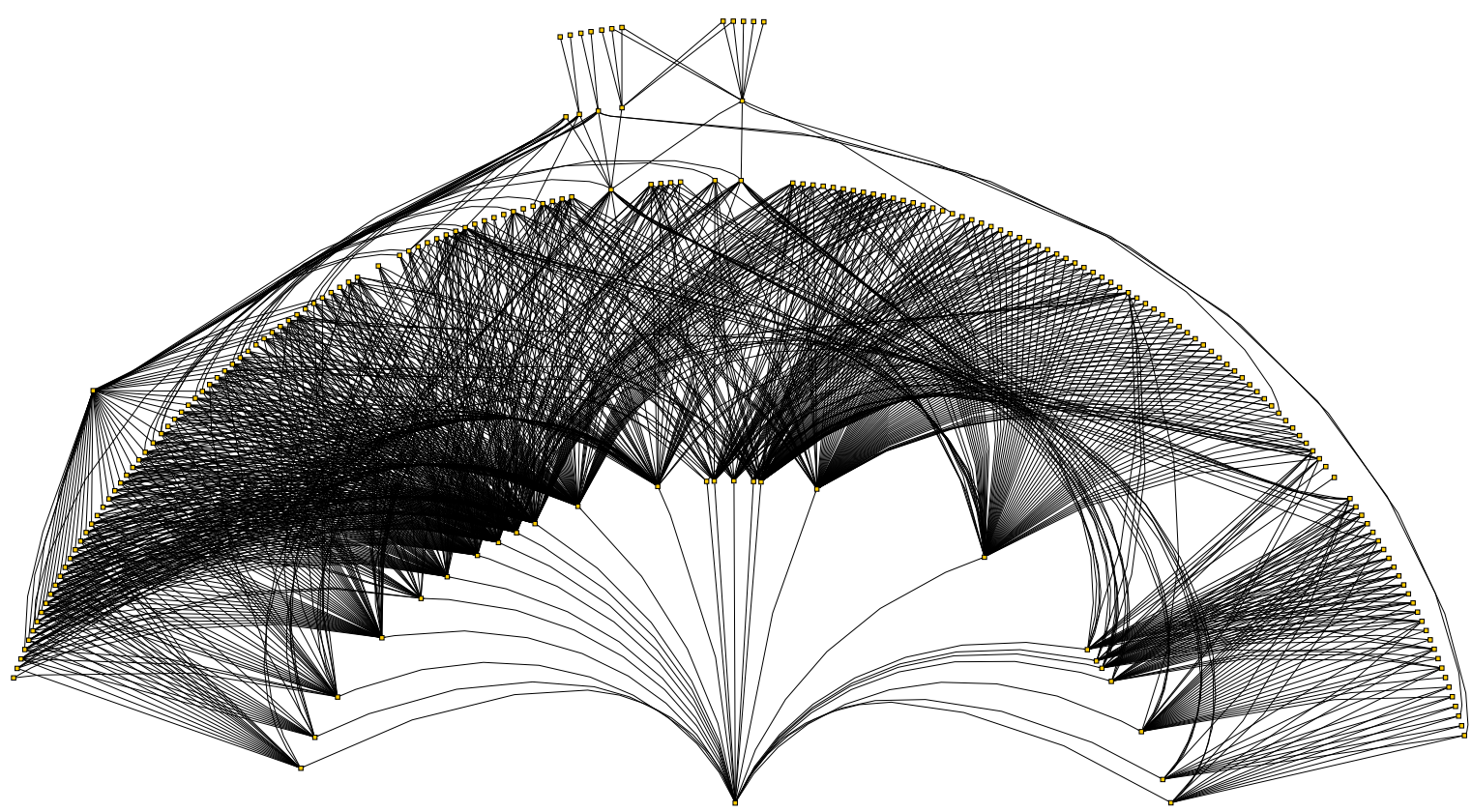

Figure 3: Construction elements (top row) and the corresponding pictures they are visible in (bottom row)

In the next step of this research, the gathered textures of construction elements will be prepared for detailed quality checks. This includes already established methods such as crack detection but also other forms of verification that material and surface match the material and geometric specifications contained in the digital model. An interest angle of analysis of this problem is that, in the context of photogrammetric reconstructions, surfaces of construction elements are typically viewed from multiple angles. This means that vision-based quality assessment can leverage results from multiple viewpoints that may be given different levels of reliability.

\section{REFERENCES}

Bosché, F. and Haas, C. (2008) 'Automated retrieval of 3D CAD model objects in construction range images', Automation in Construction, 17(4), pp. 499-512. doi: 10.1016/j.autcon.2007.09.001.

Braun, A. and Borrmann, A. (2019) 'Combining inverse photogrammetry and BIM for automated labeling of construction site images for machine learning', Automation in Construction.

Golparvar-Fard, M. et al. (2009) 'Visualization of construction progress monitoring with 4D simulation model overlaid on time-lapsed photographs', Journal of Computing ..., 23(December), pp. 391-404. doi: 10.1061/(ASCE)0887-3801(2009)23:6(391).

Nhat-Duc, H., Nguyen, Q. L. and Tran, V. D. (2018) 'Automatic recognition of asphalt pavement cracks using metaheuristic optimized edge detection algorithms and convolution neural network', Automation in Construction, 94(June), pp. 203-213. doi: 10.1016/j.autcon.2018.07.008.

Turkan, Y. et al. (2012) 'Automated progress tracking using 4D schedule and 3D sensing technologies', Automation in Construction. Elsevier B.V., 22, pp. 414-421. doi: 10.1016/j.autcon.2011.10.003. 